[13] Galárraga, L., Heitz, G., Murphy, K., Suchanek, F. M. (2014). Canonicalizing Open Knowledge Bases. Proceedings of the 23rd ACM International Conference on Conference on Information and Knowledge Management - CIKM '14. doi: https://doi.org/10.1145/2661829.2662073

[14] Bollacker, K., Evans, C., Paritosh, P., Sturge, T., Taylor, J. (2008). Freebase. Proceedings of the 2008 ACM SIGMOD International Conference on Management of Data - SIGMOD '08. doi: https:// doi.org/10.1145/1376616.1376746

[15] Shin, J., Wu, S., Wang, F., De Sa, C., Zhang, C., Ré, C. (2015). Incremental knowledge base construction using DeepDive. Proceedings of the VLDB Endowment, 8 (11), 1310-1321. doi: https://doi.org/ 10.14778/2809974.2809991

[16] Huynh, T. N., Mooney, R. J. (2008). Discriminative structure and parameter learning for Markov logic networks. Proceedings of the 25th International Conference on Machine Learning - ICML '08. doi: https://doi.org/10.1145/1390156.1390209

[17] Richardson, M., Domingos, P. (2006). Markov logic networks. Machine Learning, 62 (1-2), 107-136. doi: https://doi.org/10.1007/s10994-006-5833-1

[18] Niu, F., Zhang, C., Re, C., Shavlik, J. (2012). Scaling Inference for Markov Logic via Dual Decomposition. 2012 IEEE 12th International Conference on Data Mining. doi: https://doi.org/10.1109/icdm.2012.96

[19] Singla, P., Domingos, P. (2006). Entity Resolution with Markov Logic. Sixth International Conference on Data Mining (ICDM'06). doi: https://doi.org/10.1109/icdm.2006.65

[20] Van Haaren, J., Davis, J. (2012). Markov network structure learning: A randomized feature generation approach. Proceedings of the Twenty-Sixth AAAI Conference on Artificial Intelligence, 1148-1154.

[21] Niu, F., Ré, C., Doan, A., Shavlik, J. (2011). Tuffy. Proceedings of the VLDB Endowment, 4 (6), 373-384. doi: https://doi.org/10.14778/1978665.1978669

\title{
EXPLANATION OF THE ALGORITHMS FOR DISPLAYING 3D FIGURES ON THE COMPUTER SCREEN
}

\author{
Alexander Zhyrytovskyi \\ Institute of Computer Technologies, Graduate School \\ Open International University of Human Development "Ukraine" \\ 23 Lvivs'ka str., Kyiv, Ukraine. 04071 \\ i.am.zhirik@gmail.com
}

\begin{abstract}
Not long time ago people can only dream about the thing that they can see 3-dimensional world inside of the screen of the computer. Computers at that time can display only 256 colors on their screens, they can work only with integer numbers, and their computation speed was not fast enough. This dream comes true with the help of Id Software company. This company started to build its games by using of simplified 3D graphics. All these simplified 3D graphics were calculated by the software 3D rendering algorithms which were not published by these company. At that time there was a global goal to speedup calculations for 3D graphics and to make it look more realistic. The solution for this comes from hardware companies. Hardware companies started to work on hardware 3D accelerators, which can render 3D graphics much faster than software algorithms. These 3D accelerators are fully patented, so that no one knows how they are implemented inside. At that time there were proposed two 3D rendering interfaces: Direct3D and OpenGL. And manufacturers of graphic cards started supporting both of these interfaces. Lots of game development companies started to use these interfaces. And also Id Software company started to redesign its game engine to support these interfaces to take place in game market. From that time, everybody started to use 3D accelerators with built-in 3D rendering features and as a result everybody forgot about software 3D rendering algorithms.

Nowadays the situation come in the following way, that most of the algorithms which are used in 3D games are not published on paper, and all the 3D accelerator internal algorithms are patented. All modern 3D graphics are fully conserved by the video cards, so that 3D rendering could not be developed in other way.

The goal of this article is explanation of how $3 \mathrm{D}$ graphics can be displayed on the screen of the computer

Keywords: 3D, graphics, rendering, mesh, z-buffer, texture, projection, perspective, triangulation, triangle drawing.
\end{abstract}




\section{Introduction}

3D graphics nowadays uses in games, movies, cartoons and for engineering process. Most of things such as cars, planes, houses and portable devices before being implemented should have 3D model of itself and also 3D models of its internal parts. Therefore, 3D graphics will be in demand for the near future.

Today probably every person seen the 3-dimensional figures inside the computer, but nobody can figure out what formulas and what operations were performed to build these figures. During this article, it is possible to see all the basic steps that need to be done to build 3D figures on the screen of the computer.

\subsection{D rotation formulas}

The most fundamental part in 3D graphics is 3-dimensional rotation formulas, which looks the following:

$$
\begin{gathered}
x^{\prime}=x \cos a-y \sin a ; \\
y^{\prime}=(x \sin a+y \cos a) \cos b-z \sin b ; \\
z^{\prime}=(x \sin a+y \cos a) \sin b+z \cos b,
\end{gathered}
$$

here $\mathrm{x}, \mathrm{y}, \mathrm{z}$ - coordinates of the initial source point; $\mathrm{x}^{\prime}, \mathrm{y}^{\prime}, \mathrm{z}^{\prime}$ - coordinates of the rotated final point; $\mathrm{a}, \mathrm{b}$ - horizontal and vertical angles of the rotation.

\section{1. Flat projection}

To project 3D world point coordinates $x^{\prime}, y^{\prime}, z^{\prime}$ into the screen point coordinates $x ", y^{\prime \prime}$, it is possible to use the following formula:

$$
\begin{aligned}
& x^{\prime \prime}=x^{\prime} ; \\
& y^{\prime \prime}=y^{\prime} .
\end{aligned}
$$

Here for projection just exclude z' variable. As an example on Fig. 1, it is possible to see the figure that was previously rotated by $3 \mathrm{D}$ rotation formulas, and now it is projected down to the screen using flat projection formulas.

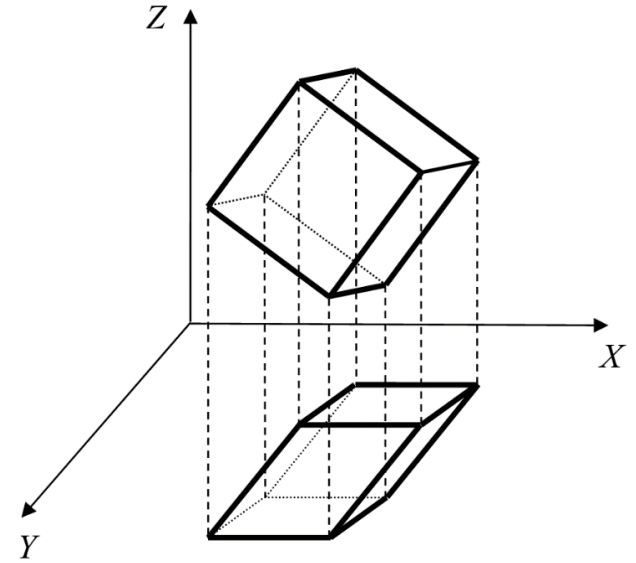

Fig. 1. Flat projection

\section{Projection with perspective}

In real world, you can look at the road and you can see that this road becomes smaller as far it is located from us (Fig. 2). 


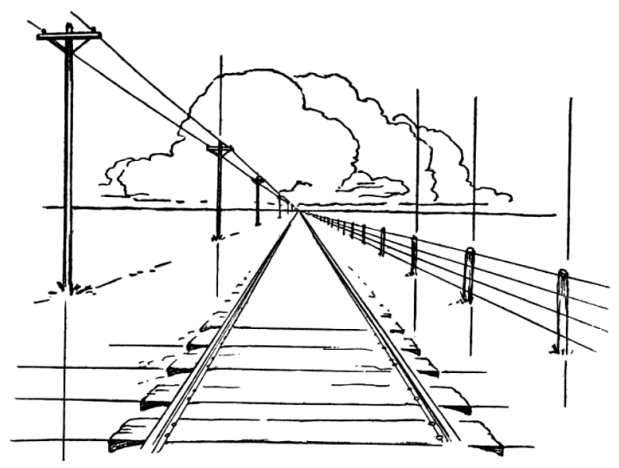

Fig. 2. Perspective

In some distance from us, the object becomes really small so that we stop seeing it. The distance from the screen to the center of perspective comes here as value of perspective (Fig. 3).

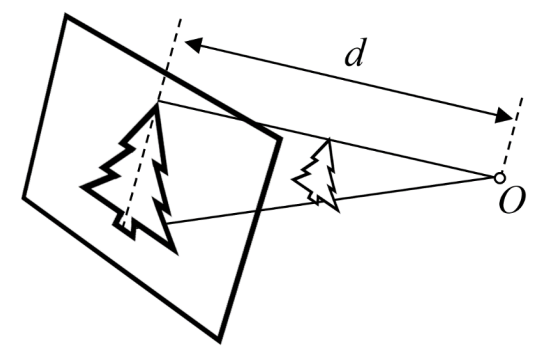

Fig. 3. Projection with perspective (as far the object locates from us, as smaller it is)

Such projection is described using following formulas:

$$
\begin{aligned}
& x^{\prime \prime}=x^{\prime} \frac{d}{d+z^{\prime}} ; \\
& y^{\prime \prime}=y^{\prime} \frac{d}{d+z^{\prime}},
\end{aligned}
$$

here $x^{\prime}, y^{\prime}, z^{\prime}$ - coordinates of the initial source point; $x ", y^{\prime \prime}$ - coordinates of the final rotated point; $d$-value of perspective.

\section{Drawing 3D figure using dots}

The simplest 3D figures can be built using dot-by-dot projection to the screen. The example of such figure is displayed on Fig. 4.

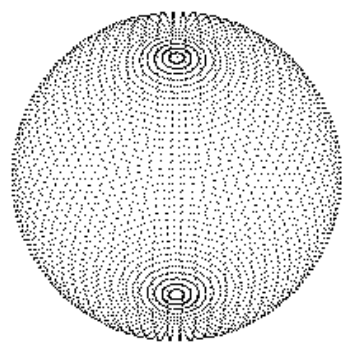

Fig. 4. Drawing 3D figure using dots

\section{1. Drawing 3D figure using mesh}

Other approach is to use lines instead of dots. In this case, all the projected points we can connect into mesh of lines (Fig. 5). 


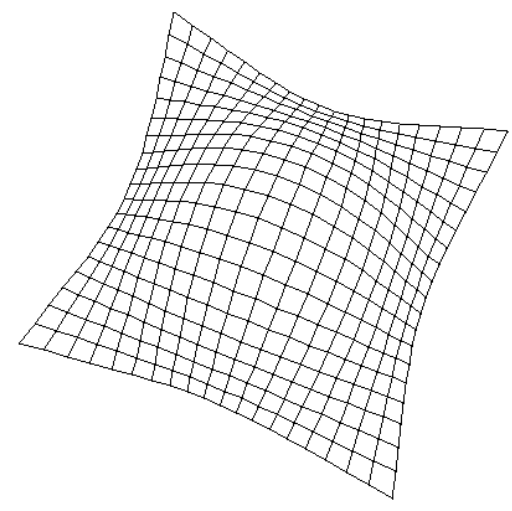

Fig. 5. Drawing 3D figure using mesh

\section{2. Triangulation}

To make the figure solid it is possible to build it using mesh of solid triangles (Fig. 6).

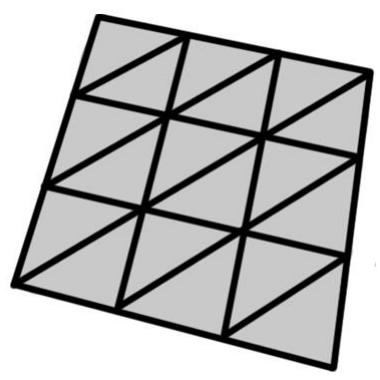

Fig. 6. Solid triangle-based mesh

\section{3. Triangle drawing algorithm}

To draw the triangle itself, there is triangle drawing algorithm. On Fig. 7, it is possible to see how triangle drawing algorithm works.

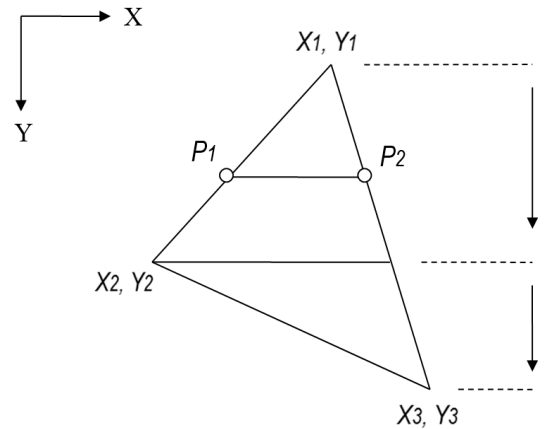

Fig. 7. Triangle drawing algorithm

Algorithm:

1. Sort triangle vertices so that: $\mathrm{Y}_{1} \leq \mathrm{Y}_{2} \leq \mathrm{Y}_{3}$;

2. Create two points $\mathrm{P}_{1}$ and $\mathrm{P}_{2}$ (Fig. 7). At the beginning both of these points will have coordinates $\left(\mathrm{X}_{1}, \mathrm{Y}_{1}\right)$;

3. Calculate horizontal increments $\mathrm{d}_{1}$ and $\mathrm{d}_{2}$, and also number of iterations $\mathrm{N}$. It is possible to do this by the following formulas:

$$
\mathrm{d}_{1}=\frac{\mathrm{X}_{2}-\mathrm{X}_{1}}{\mathrm{Y}_{2}-\mathrm{Y}_{1}}
$$




$$
\begin{gathered}
d_{2}=\frac{X_{3}-X_{1}}{Y_{3}-Y_{1}} ; \\
N=Y_{2}-Y_{1} .
\end{gathered}
$$

1. Repeat it $\mathrm{N}$ times: move the points $\mathrm{P}_{1}$ and $\mathrm{P}_{2}$ by the one step to the bottom. Also, it is necessary to move horizontally $\mathrm{P}_{1}$ by the increment $\mathrm{d}_{1}$. And also to move horizontally $\mathrm{P}_{2}$ by the increment $\mathrm{d}_{2}$. And fill empty horizontal place between $\mathrm{P}_{1}$ and $\mathrm{P}_{2}$ using dots;

2. Recalculate first increment $d_{1}$ and number of iterations $\mathrm{N}$ :

$$
\begin{gathered}
\mathrm{d}_{1}=\frac{\mathrm{X}_{3}-\mathrm{X}_{2}}{\mathrm{Y}_{3}-\mathrm{Y}_{2}} ; \\
\mathrm{N}=\mathrm{Y}_{3}-\mathrm{Y}_{2} .
\end{gathered}
$$

3. Repeat it $\mathrm{N}$ times: move the points $\mathrm{P}_{1}$ and $\mathrm{P}_{2}$ by the one step to the bottom. Also it is necessary to move horizontally $\mathrm{P}_{1}$ by the increment $\mathrm{d}_{1}$. And also to move horizontally $\mathrm{P}_{2}$ by the increment $\mathrm{d}_{2}$. And fill empty horizontal place between $\mathrm{P}_{1}$ and $\mathrm{P}_{2}$ using dots.

\section{4. Z-buffer}

When drawing solid triangle mesh on the screen if these triangles are collapsed, it is possible to see visual ordering problem (Fig. 8).

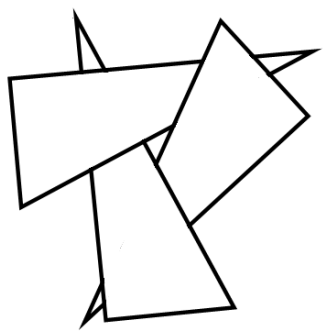

Fig. 8. Triangle order displaying problem

Basically this problem can happen if on the same place we need to draw 2 points with different colors and different z-distances. And the problem is how to keep the point with minimal z-distance. To solve this problem, it is necessary to use extra image which will contain z-distances for all the points that are displayed on the screen, this image is called z-buffer, it is possible to see it on Fig. 9.

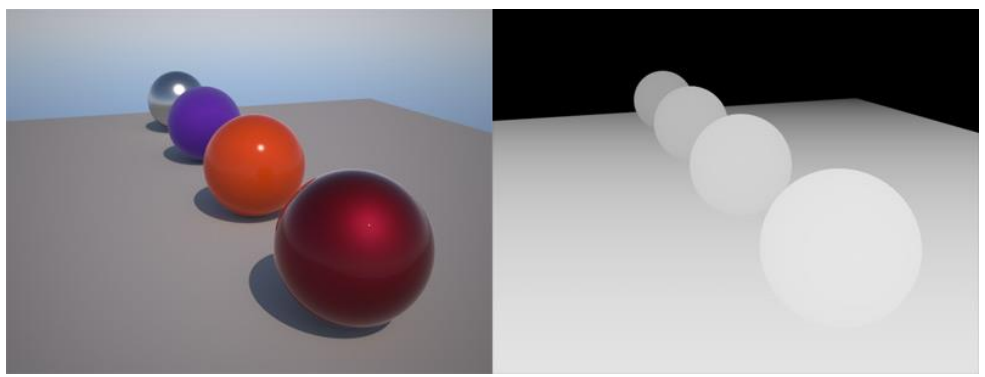

Fig. 9. Normal image and z-buffer image

Let's suppose that at the beginning z-buffer filled with extremely big numbers. When we want to put a point on the screen, we can do this only if its z-distance is smaller than z-distance of corresponding point of z-buffer. After when we put the point on the screen we also need to put its $\mathrm{z}$-distance to $\mathrm{z}$-buffer. 


\section{Filling the triangle with 3 color gradient}

Triangle has 3 vertices. In this approach, each triangle vertex has its coordinates and color. To find the color of the current point inside triangle it is necessary to find the distances to each of the vertices (Fig. 10).
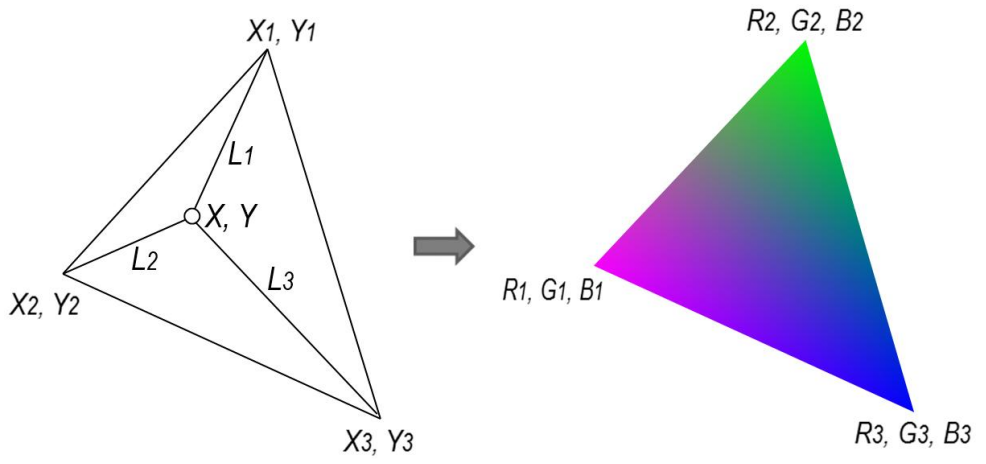

Fig. 10. Filling triangle with 3 color gradient

Let's find these distances by the following formulas:

$$
\begin{aligned}
& \mathrm{L}_{1}=\sqrt{\left(\mathrm{X}-\mathrm{X}_{1}\right)^{2}+\left(\mathrm{Y}-\mathrm{Y}_{1}\right)^{2}} ; \\
& \mathrm{L}_{2}=\sqrt{\left(\mathrm{X}-\mathrm{X}_{2}\right)^{2}+\left(\mathrm{Y}-\mathrm{Y}_{2}\right)^{2}} ; \\
& \mathrm{L}_{3}=\sqrt{\left(\mathrm{X}-\mathrm{X}_{3}\right)^{2}+\left(\mathrm{Y}-\mathrm{Y}_{3}\right)^{2}},
\end{aligned}
$$

here $\mathrm{X}, \mathrm{Y}$ - coordinates of current point inside triangle; $\mathrm{X}_{1}, \mathrm{Y}_{1}$ - coordinates of first vertex of the triangle; $\mathrm{X}_{2}, \mathrm{Y}_{2}$ - coordinates of second vertex of the triangle; $\mathrm{X}_{3}, \mathrm{Y}_{3}$ - coordinates of third vertex of the triangle; $\mathrm{L}_{1}, \mathrm{~L}_{2}, \mathrm{~L}_{3}$ - distances from current point to each of vertices. formulas:

Using these distances, it is possible to calculate the color of current point by the following

$$
\begin{aligned}
& R=\frac{R_{1} \cdot L_{1}+R_{2} \cdot L_{2}+R_{3} \cdot L_{3}}{L_{1}+L_{2}+L_{3}} ; \\
& G=\frac{G_{1} \cdot L_{1}+G_{2} \cdot L_{2}+G_{3} \cdot L_{3}}{L_{1}+L_{2}+L_{3}} ; \\
& B=\frac{B_{1} \cdot L_{1}+B_{2} \cdot L_{2}+B_{3} \cdot L_{3}}{L_{1}+L_{2}+L_{3}} ;
\end{aligned}
$$

here $R_{1}, G_{1}, B_{1}$ - red, green and blue components of the vertex 1 color; $R_{2}, G_{2}, B_{2}$ - red, green and blue components of the vertex 2 color; $R_{3}, G_{3}, B_{3}$ - red, green and blue components of the vertex 3 color; R, G, B - red, green and blue components of the destination color for the current point.

\section{1. Texture overlay on 3D figure}

Texture overlaying on triangle is very similar to filling it with 3 color gradient, the only difference is that instead color component variables it is necessary to use texture coordinate variables (Fig. 11).

To draw 3D figure with texture overlay, first wit is necessary to take some texture image and divide it into triangles. This diving means that for each triangle we need to calculate its coordinates on texture (Fig. 12). 


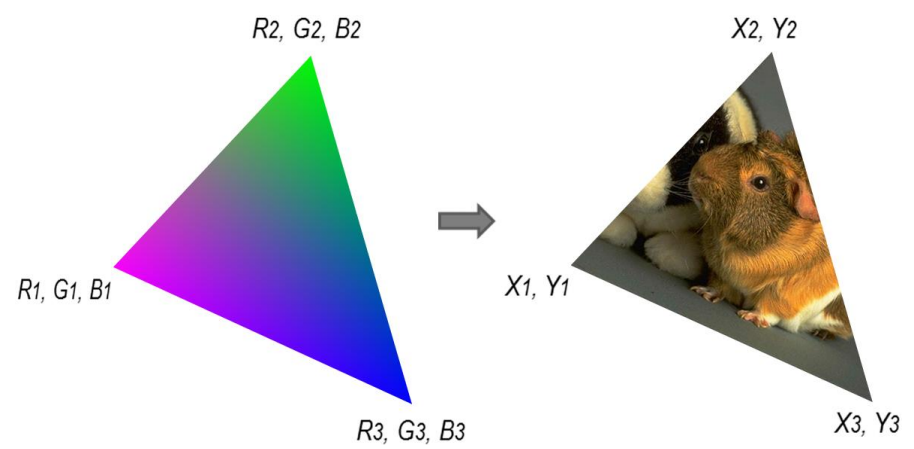

Fig. 11. Drawing texture on the triangle

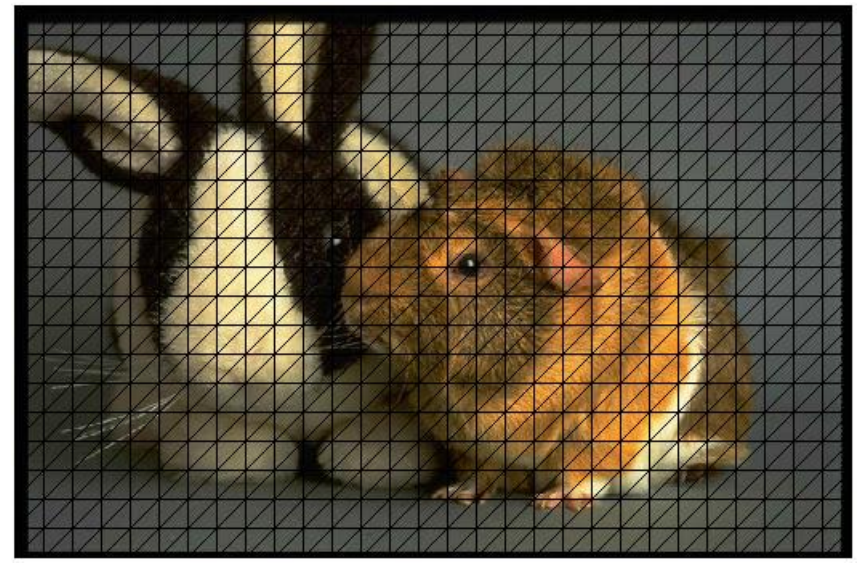

Fig. 12. Overlaying the texture over the mesh of triangles

After that, it is possible to change coordinates of the mesh with triangles, and produce any 3D figure with texture (Fig. 13).
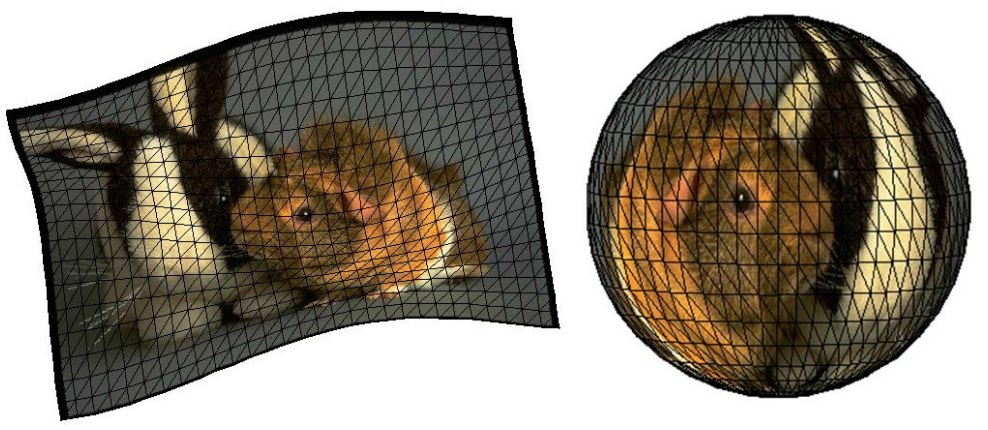

Fig. 13. Wrapping mesh of triangles into different shapes

\section{Conclusion}

Theoretical algorithms for building 3D figures, that were provided in this article, were implemented and tested. It is possible to see their implementation in software program, which can be viewed by the following link: http://alex.zhyrytovskyi.x10.name/progs/toys/texture3D.zip

As the result using these algorithms, it is possible to build hardware independent 3D graphics which can be calculated in real time without usage of any graphic card interfaces such as DirectX or OpenGL.

Also nowadays, there looks to be no literature that describing $3 \mathrm{D}$ rendering process, and possibly this article can open eyes of the reader on 3D rendering process and will help him to understand how it performs. 


\section{References}

[1] Ammeraal, L. (1986). Programming Principles in Computer Graphics. New York: Wiley, 168.

[2] Angel, E., Shreiner, D. (2012). Interactive Computer Graphics: A Top-Down Approach with OpenGL. Addison-Wesley.

[3] Botsch, M., Kobbelt, L., Pauly, M,. Alliez, P., Lévy, B. (2010). Polygon Mesh Processing. A K Peters/CRC Press, 250. doi: https://doi.org/10.1201/b10688

[4] Glaeser, G. (1994). Fast Algorithms for 3D-Graphics. Springer-Verlag, 306. doi: https:// doi.org/10.1007/978-3-662-25798-2

[5] Hearn, D., Baker, M. P. (1994). Computer Graphics. Prentice Hall.

[6] Horowitz, E., Sahni, S., Mehta, D. (1995). Fundamentals of Data Structures in C++. Computer Science Press, New York.

[7] Kernighan, B., Ritchie, D. (1988). The C Programming Language. Prentice Hall, Englewood Cliffs NJ.

[8] Lafore, R. (1991). Object-Oriented Programming in Turbo C++. Waite Group Press, Mill Valley CA.

[9] Laszlo, M. J. (1996). Computational Geometry and Computer Graphics in C++. Prentice Hall, Eaglewood Cliffs NJ.

[10] Luse, M. (1993). Bitmapped Graphics Programming in C++. Addison-Wesley, Reading MA.

[11] Lippman, S. B. (1991). C++ Primer. Addison-Wesley, Reading MA, 630.

[12] Mortenson, M. E. (1989). Computer Graphics: An Introduction to the Mathematics and Geometry. Industrial Press, New York.

[13] Norling, E. R. (1939). Perspective Made Easy. Mineola, 228.

[14] Porev, V. (2002). Kompiuternaia hrafyka. Sankt-Peterburg: BHV-Peterburg.

[15] Kvicinskiy, E. Triangle drawing algorithm. Available at: http://grafika.me/node/67

[16] Z-buffer channel. Available at: http://support.nextlimit.com/display/maxwell4/Z-buffer+ channel 